\title{
A99 HETEROGENEOUS NUCLEAR RIBONUCLEOPROTEIN- ASSOCIATED NUCLEIC ACIDS TRIGGER DISEASE IN A RAT TRANSFER MODEL OF ARTHRITIS
}

MHoffmann, CW Steiner, C Baumann, SHerman, GSteiner Division of Rheumatology, Medical University of Vienna, Austria

\subsection{6/ard.2010.129627|}

Toll-like receptor (TLR)-mediated autoimmunity to nucleic acid-protein complexes has been implicated in the pathogenesis of systemic lupus erythematosus, whereas information regarding the influence of nucleic acid sensing TLRs on the development of rheumatoid arthritis (RA) is scarce. The authors have recently shown that the nucleic acid-binding autoantigen heterogeneous nuclear ribonucleoprotein (hnRNP)-A2 is not only a preferred $B$ and $T$ cell autoimmune target in pristane-induced arthritis (PIA), but is also able to induce a MyD88-dependent secretion of proinflammatory cytokines from rat or murine splenocytes. ${ }^{1}$

The authors now show that experimental arthritis induced by intradermal injection of the isoprenoid alkane pristane into rats can be transferred to naïve recipient rats with pristaneprimed splenocytes restimulated with hnRNP-A2 or related hnRNP proteins. Remarkably, disease could also be transferred with splenocytes incubated with RNA and/or DNA sequences known to bind to hnRNPs. The transferred disease clinically and histologically resembles arthritis triggered by direct injection of pristane. Disease transfer could be inhibited with chloroquine and was also achieved by stimulation of splenocytes with imidazoquinolines and/or CpG DNA. Transfer of arthritis was mediated by CD4-positive $\mathrm{T}$ cells and required co-incubation with TLR7- and/or TLR9-expressing antigen-presenting cells such as plasmacytoid dendritic cells (pDC). $\mathrm{pDC}$ reacted to in vitro stimulation with hnRNPs with a strong secretion of type I interferons and an upregulation of TLR7 mRNA.

Taken together, these data strongly suggest that nucleic acidassociated autoantigens such as hnRNPs can play a primary role in the induction of arthritogenic autoimmunity in PIA and, possibly, also in human RA where hnRNP proteins are known to be targets of the patients' autoimmune response.

\section{REFERENCE}

1. Hoffmann et al. J Immunol 2007. 